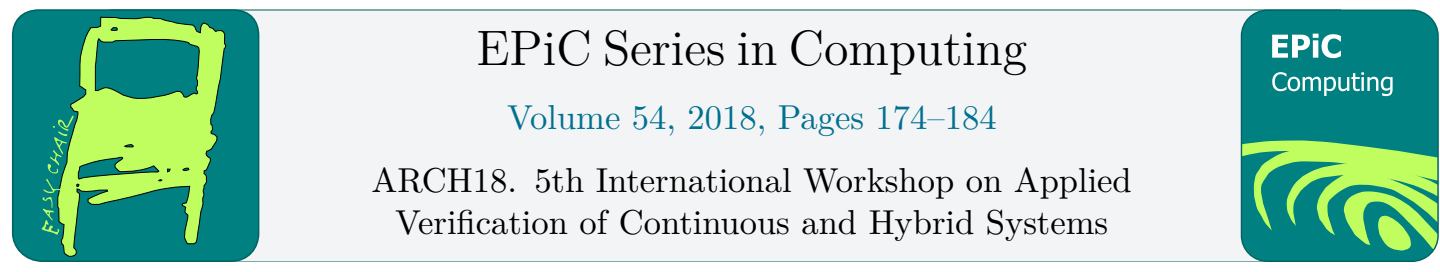

\title{
Linear Differential-Algebraic Equations (Benchmark Proposal)
}

\author{
Patrick Musau, Diego Manzanas Lopez, Hoang-Dung Tran, and Taylor T. \\ Johnson \\ Vanderbilt University, Nashville, Tennessee, USA \\ patrick.musau@vanderbilt.edu, diego.manzanas.lopez@vanderbilt.edu, \\ dung.h.tran@vanderbilt.edu, taylor.johnson@vanderbilt.edu
}

\begin{abstract}
This benchmark suite consists of eight small-to-large scale index-1 to index-3 linear differential algebraic systems (DAEs) derived from various application domains in engineering and science that exemplify the systemic prevalence of DAE systems in cyber-physical system applications. While in the last two decades numerous verification approaches and tools have been developed for systems described by ordinary differential equations, there is currently a lack of research methods for differential algebraic equations. Thus, the verification of DAE systems remains an open problem that has not been adequately addressed in the research literature. The following paper seeks to address this shortcoming by presenting a series of benchmark problems to stimulate the development of efficient and scalable tools for DAE verification and falsification. The benchmark models presented in this manuscript are available in the SpaceEx format using a tool named Daev for model generation.
\end{abstract}

Category: Academic Difficulty: Hard

\section{Context and Origins}

Reachability analysis plays a fundamental role in verifying and falsifying cyber-physical systems (CPS) in numerous computer-assisted formal analysis regimes. The operation of CPS is governed by physical laws typically described by a set of differential equations. In recent years, there have been numerous tools and approaches developed for a class of systems described by ordinary differential equations (ODEs). In this context, given an initial set of states, we typically wish to compute an over-approximation of the reachable states in a given time period using techniques such as zonotopes [1,18], support functions [19], Taylor methods [10], the generalized star set $[4,14]$, and various other set representations. While there are numerous verification approaches for ODEs, there is a lack of methodology in dealing with systems described by differential algebraic equations (DAEs). Differential algebraic equations express a more general class of dynamics than ODEs and are extensively used to describe numerous problems in engineering and science, such as multi-body mechanics, electrical circuit design, chemical process simulation, heat and gas transfer, neural network modeling, atmospheric physics, thermodynamic 
systems, and water distribution networks [7]. In this domain, a differential equation describing the dynamics of the system is coupled with algebraic constraints expressing restrictions on the system's allowable behaviors. These restrictions typically include conservation laws, position constraints, circuit laws, or mass and entropy balance equations [22].

The verification of DAE systems is significantly more complex than for ODE systems, especially for high index DAEs, since they are described by both differential and algebraic equations. Whereas sufficient continuity is enough to obtain a unique solution to an ordinary differential equation, the theory of existence and uniqueness for DAEs depends heavily upon their structure. Additionally, often the solution of a DAE depends on the derivative of an input signal where this is not the case for ODEs [22]. Thus, in order to perform reachability analysis on a DAE system, it must first be decoupled into an ODE subsystem and one or several algebraic constraints. Additionally, unlike in ODE verification, where the user is freely allowed to specify an initial set of states, in order to guarantee a solution for a DAE, the initial states and inputs of a system must be consistent and satisfy certain conditions [16]. Thus, we must employ consistency checking methods for the initial set in order to compute the reachable set of a DAE system.

To the best of the authors' knowledge, there are only a few existing verification frameworks for DAE systems and the majority of these frameworks deal with DAE systems of index$1[11,13,25]$. Additionally, most of these regimes make use of level set methods in which the reachability problem is reformulated into solving the Hamilton-Jacobi partial differential equation by discretizing the state space [2]. The major drawback of these verification schemes is their lack of scalability with respect to industry-relevant applications since the computational complexity of these methods is exponential with respect to the number of state variables [2]. Thus, in an effort to address the scalability issues experienced in DAE verification schemes, Matthias Althoff et al. presented a zonotope-based verification algorithm for non-linear, semiexplicit index-1 DAE systems [2] capable of dealing with 42 continuous state variables. In another work by Dang et al. [13], the authors make use of polyhedral set representations for DAEs in electrical circuits. While their techniques scale far better than level set methods they are not as efficient as those presented in [2]. While these research efforts serve as a preliminary step towards efficient DAE verification, there is an urgent need for methods and tools that can deal with DAEs of higher index due to the large number of CPS applications that involve high index DAE dynamics $[3,6,26]$.

Inspired by the lack of verification methods for high-index DAEs in the research literature, the central contribution of this paper is the provision of a series of executable and scalable benchmark problems for linear DAE systems of up to index-3. The set of benchmarks that we present in this paper are widely used in practice and will be useful in the development of novel reachability methods for the verification and falsification of DAE CPS. The problems that we consider exemplify a wide range of problems in electronic circuits, physics, and fluid dynamics with up to $n=4880$ state variables. Conversion of the benchmark suite into a format that can be analyzed by the available verification tools is non-trivial, and in our regime we must first decouple the DAE system and perform a consistency check of its initial set of states and inputs. Once the DAE is decoupled and the consistency of its parameters is ensured, we can construct an automaton using the ODE subsystem and express the algebraic constraints as a set of invariants derived from the decoupling process. This conversion and printing into the SpaceEx [15] input format is implemented in a tool called Daev ${ }^{1}$, that makes use of Python and

\footnotetext{
${ }^{1}$ The benchmark problems and software tool Daev are available online at: https://github.com/verivital/ daev/tree/master/daev
} 
its standard packages Numpy, Scipy, and Mathplotlib. A detailed description of the software tool Daev, discussion of decoupling techniques, and simulation of the benchmarks described in this paper can be found in [29]. Currently, the only DAEs that can be analyzed by the tools available in the research community are of index-1.

\section{Description of Benchmarks}

The general form of a linear DAE system is given by:

$$
\begin{aligned}
\mathbf{E} \dot{\mathbf{x}}(t) & =\mathbf{A} \mathbf{x}(t)+\mathbf{B u}(t), \\
\mathbf{y}(t) & =\mathbf{C}^{T} \mathbf{x}(t)+\mathbf{D}^{T} \mathbf{u}(t)
\end{aligned}
$$

where $\mathbf{x}(t) \in \mathbb{R}^{n}$ is the state vector of the system, $\mathbf{E}, \mathbf{A} \in \mathbb{R}^{n \times n}, \mathbf{B} \in \mathbb{R}^{n \times m}, \mathbf{C} \in \mathbb{R}^{n \times l}$, and $\mathbf{D} \in \mathbb{R}^{m \times l}$ are the system's coefficient matrices in which $\mathbf{E}$ is singular, and $\mathbf{u}(t) \in \mathbb{R}^{m}, \mathbf{y}(t) \in \mathbb{R}^{l}$ are the input and output of the system respectively.

Bearing the above in mind, in this section we present a brief description of the benchmark problems for DAE verification. Table 5.1 summarizes the DAE index, number of variables, and safety specifications for each benchmark. A complete description of the initial conditions and inputs for each benchmark can be found in the model files included in the supplementary material for this paper.

\section{$2.1 \quad$ RL Network}

In circuit analysis, the dynamics of many resistor networks can be characterized by DAEs. Typically, this results from the use of the modified nodal analysis method (MNA), which characteristically yields a DAE. [5]. In this problem, we consider a simple RL network and wish to determine the current through the inductor as well as the node voltages for the system shown in Figure 2.1. The system matrices, state vector, and input function are given by the following

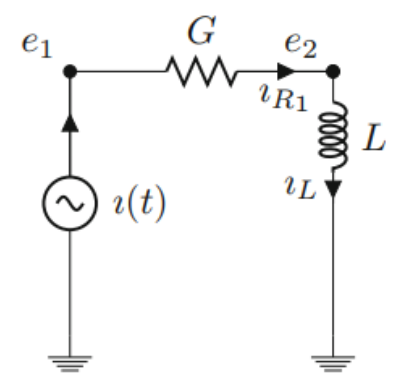

Figure 2.1: Simple RL network as given in [5]

index-2 DAE:

$$
\begin{aligned}
\mathbf{E} \dot{\mathbf{x}}(t) & =\mathbf{A} \mathbf{x}(t)+\mathbf{B} \mathbf{u}(t), \quad \mathbf{x}(0)=\mathbf{x}_{\mathbf{0}} \\
\mathbf{y}(t) & =\mathbf{C}^{T} \mathbf{x}(t)
\end{aligned}
$$


where $\mathbf{u}(t)=\iota(t), \mathbf{x}(t)=\left[e_{1}, e_{2}, \iota_{L}\right]$

$$
\mathbf{E}=\left[\begin{array}{lll}
0 & 0 & 0 \\
0 & 0 & 0 \\
0 & 0 & L
\end{array}\right] \quad \mathbf{A}=\left[\begin{array}{ccc}
-G & G & 0 \\
G & -G & -1 \\
0 & 1 & 0
\end{array}\right] \quad \mathbf{B}=\left[\begin{array}{l}
1 \\
0 \\
0
\end{array}\right] \quad \mathbf{C}=\mathbf{B} .
$$

In this benchmark, we consider two safety specifications related to value of the node voltages. The first specification seeks to determine whether the node voltage $e_{1}$ and $e_{2}$ drops below $-0.2 \mathrm{~V}$ and $-0.1 \mathrm{~V}$ respectively. The second specification seeks to verify whether the node voltage $e_{2}$ remains above $0.2 \mathrm{~V}$ at all times.

\section{$2.2 \quad$ RLC Circuit}

The RLC circuit benchmark consists of a simple circuit network described in [12]. The equations characterizing the circuit are given by:

$$
\left[\begin{array}{cccc}
L & 0 & 0 & 0 \\
0 & 0 & 1 & 0 \\
0 & 0 & 0 & 0 \\
0 & 0 & 0 & 0
\end{array}\right]\left[\begin{array}{c}
\dot{I}(t) \\
\dot{V}_{L}(t) \\
\dot{V}_{C}(t) \\
\dot{V}_{R}(t)
\end{array}\right]=\left[\begin{array}{cccc}
0 & 1 & 0 & 0 \\
\frac{1}{C} & 0 & 1 & 0 \\
-R & 0 & 0 & 0 \\
0 & 1 & 1 & 1
\end{array}\right]\left[\begin{array}{c}
I(t) \\
V_{L}(t) \\
V_{C}(t) \\
V_{R}(t)
\end{array}\right]+\left[\begin{array}{c}
0 \\
0 \\
0 \\
-1
\end{array}\right] V_{s}(t)
$$

where the voltage source $V_{s}(t)$ is the control input, and $V_{R}(t), V_{L}(t), V_{C}(t)$ denote the voltage across the resistor, inductor, and capacitor respectively. In this benchmark, we consider the following safety specifications: the sum of value of the current in the circuit loop and the value of the voltage across the resistor should have a value greater than $0.2 \mathrm{~V}$. The second specification requires that the voltage across the resistor remains below $-0.3 \mathrm{~V}$ at all times.

\subsection{Electrical Generator}

The following benchmark deals with a time-invariant model of an electrical generator presented in [17]. The model is characterized by the following parameters as shown in Figure 2.2:

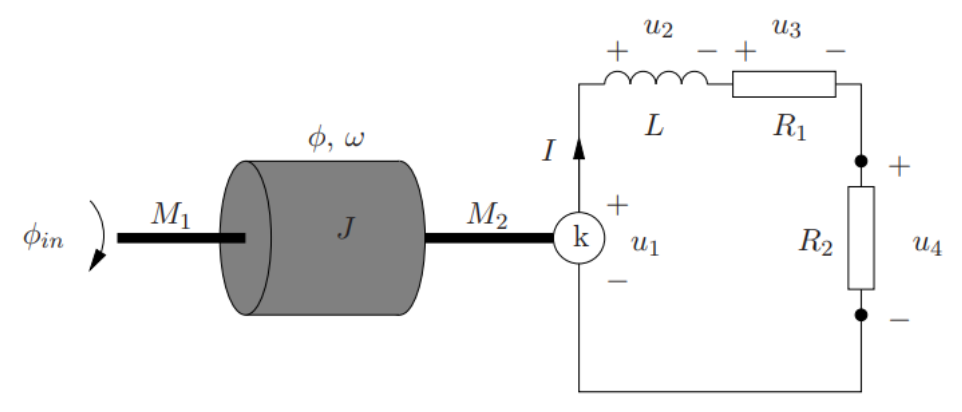

Figure 2.2: Model of electrical generator as presented in [17]

where $\phi_{i n}$ is the input angle on the left axis, $\phi$ denotes the angle with which the mass is rotated with respect to the axis, $\omega$ is the angular velocity, and $M 1$ and $M 2$ represent the torques on the 
right and left side of the mass respectively. The rotating mass is then connected to the generator on another axis which is composed of resistors and an inductor. The current-dependent value of the torque is given by $M 2=k I$ and the voltage across the connection point, $k$, is given by the relation $u_{1}=k \omega$. In this problem, the quantity that we are concerned with is the voltage across the resistor $R_{2}$ [17]. The system equations are given by:

$$
\begin{array}{ccc}
\phi_{\text {in }}=\phi & \dot{\phi}=\omega & J \dot{\omega}=M_{1}+M_{2} \\
M_{2}=k I & u_{1}=k \omega & u_{2}=L \dot{I} \\
u_{3}=R_{1} I & u_{4}=R_{2} I & u_{1}=u 2+u_{3}+u_{4}
\end{array}
$$

The two safety specifications we considered in this problem were as follows: the first determined if the voltage across the resistor $R_{2}$ remained above $0.01 \mathrm{~V}$, and the second specification determined if the torque had a value of $1.0 \mathrm{~N} \cdot \mathrm{m}$ or greater.

\subsection{Damped Mass}

In this benchmark, we consider a holonomically constrained damped mass-spring system. The system is constructed by connecting the $i$ - $t h$ mass of weight $m_{i}$ to mass $m_{i+1}$ with an additional connection to the ground using a spring and a damper (one of each is employed for each connection). The damper and spring constants are $\delta_{i}$ and $d_{i}, k_{i}$ and $\kappa_{i}$ respectively. The only exception to the described configuration is that the first mass is connected to the last mass, and it is this mass that we control. Figure 2.3 displays the architecture of the damped mass-spring system. The vibrations of the system can be described by the following equations [20]:

$$
\begin{aligned}
\dot{\mathbf{p}}(t) & =\mathbf{v}(t), \\
M \dot{\mathbf{v}}(t) & =K \mathbf{p}(t)+D \mathbf{v}(t)-G^{T} \lambda(t)+B_{2} u(t), \\
0 & =G \mathbf{p}(t), \\
y(t) & =C_{1} \mathbf{p}(t)
\end{aligned}
$$

where $\mathbf{p}(\mathrm{t}) \in \mathbb{R}^{g}$ is the position vector, $\mathbf{v}(\mathrm{t}) \in \mathbb{R}^{g}$ is the velocity vector, $\lambda(t) \in \mathbb{R}^{2}$ is the Lagrange multiplier, $M=\operatorname{diag}\left(m_{1}, \ldots, m_{g}\right)$ is the mass matrix, $D$ and $K$ are the tridiagonal damping and stiffness matrices, $G=[1,0, \ldots, 0,-1] \in \mathbb{R}^{1, g}$ is the constraint matrix, $B_{2}=e_{1}$ and $C_{1}=\left[e_{1}, e_{2}, e_{s-1}\right]^{T}$. $e_{i}$ denotes the $i$-th column of the identity matrix $I_{g}$. The DAE describing this system is of index-3 and in this system we considered two safety specifications relating to the position of the masses. These specifications can be found in Table 5.1.

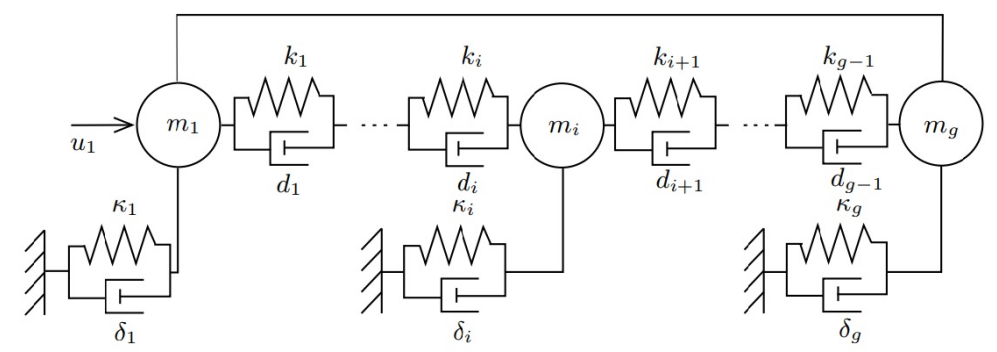

Figure 2.3: Architecture of a damped mass-spring system with a holonomic constraint as presented in [20] 


\subsection{Partial Element Equivalent Circuit (PEEC)}

In electromagnetic circuit analysis, the partial element equivalent circuit method allows one to solve problems in which there is an electromagnetic part as well as a circuit portion [27]. As an example, the method can be employed for the calculation of inductance in interconnected problems. This approach is a full wave electromagnetic electrical procedure for embedded circuits including conductors in arbitrary dielectrics in terms of equivalent circuits [27]. As a result, these models are primarily used in two domains: time and frequency.

The PEEC model used in this paper is an LC circuit model of a patch antenna structure containing 2100 capacitances, 172 inductances and 6990 mutual inductances. The set up of the system considered in this paper contains 480 state variables [9]. This benchmark problem was originally proposed by Chahloui et al. to test numerical methods for order reduction abstraction techniques and we refer readers to this paper for further detail [8].

\subsection{Modified Nodal Analysis (MNA)}

The nodal analysis method has been widely utilized to describe and solve circuit equations in computer-aided network analysis. However, despite its success, this method has several limitations, including the inefficient treatment of voltage sources, the inability to include currentdependent elements, and a lack of accuracy in obtaining branch currents as part of the output of the program [21]. Therefore, a modified nodal analysis approach (MNA) has been developed to overcome these problems. The modified nodal analysis approach (MNA) can be written in general form as a system of differential algebraic equations [9]:

$$
\begin{aligned}
E \dot{\mathbf{x}}_{\mathbf{n}} & =A \mathbf{x}_{\mathbf{n}}+\mathbf{B} \mathbf{u}_{\mathbf{p}} \\
\dot{\mathbf{i}}_{\mathbf{p}} & =C \mathbf{x}_{\mathbf{n}}
\end{aligned}
$$

The $i_{p}$ and $u_{p}$ terms correspond to the port current and voltages respectively. The matrices of equation 2.7 can be written as

$$
\mathbf{A}=\left[\begin{array}{cc}
-N & -G \\
G^{T} & 0
\end{array}\right] \mathbf{E}=\left[\begin{array}{cc}
L & 0 \\
0 & H
\end{array}\right] \mathbf{x}_{\mathbf{n}}=\left[\begin{array}{c}
v \\
i
\end{array}\right]
$$

where $v$ and $i$ are the MNA variables corresponding to the node, inductor, and source voltages respectively. $\mathbf{E} \in \mathbb{R}^{n \times n}$ is a singular matrix that corresponds to the susceptance matrix, and $A \in \mathbb{R}^{n \times n}$ is the conductance matrix. $\mathrm{N}, \mathrm{L}$, and $\mathrm{H}$ are the matrices containing the values of the resistors, capacitors and inductors, respectively. Finally, G represents the current variables in KCL equations, whose values can only be $-1,0$, or 1 . In this benchmark, the safety specification that we considered was related to the the node voltages. In particular, we required the voltage of node1 to be greater than $-0.001 \mathrm{~V}$.

\subsection{Interconnected Rotating Mass}

The interconnected rotating mass system consists of two rotating masses connected on a rotational axis. The system is described by the torques $M_{1}, M_{2}, M_{3}$, and $M_{4}$, and by angular velocities $z_{1}$ and $z_{2}$.

The dynamics of the system are described by: 


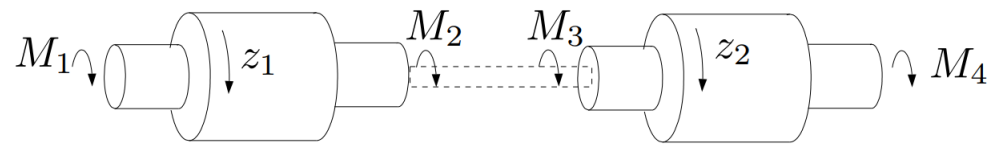

Figure 2.4: Structure of two rotating interconnected masses as presented in [28]

$$
\begin{aligned}
& J_{1} z_{1}=M_{1}+M_{2}, \\
& J_{1} z_{1}=M_{1}+M_{2}, \\
& M_{2}=-M_{3}, \\
& z 1=z 2
\end{aligned}
$$

We can write the dynamics of the system as a DAE system in standard form, obtaining the following equations as a result:

$$
\left[\begin{array}{cccc}
1 & 0 & 0 & 0 \\
0 & 0 & 0 & 0 \\
0 & 0 & 0 & 0 \\
0 & \frac{J_{1} J_{2}}{J_{1}+J_{2}} & 0 & 0
\end{array}\right] \dot{\mathbf{z}}+\left[\begin{array}{cccc}
0 & 0 & 0 & 0 \\
0 & 1 & 0 & 0 \\
0 & 0 & 1 & 0 \\
0 & 0 & 0 & 1
\end{array}\right] \mathbf{z}=\left[\begin{array}{cc}
1 & 1 \\
0 & 0 \\
0 & 0 \\
\frac{J_{2}}{J_{1}+J_{2}} & \frac{-J_{1}}{J_{1}+J_{2}}
\end{array}\right]\left[\begin{array}{c}
M_{1} \\
M_{2}
\end{array}\right]
$$

\subsection{Semi-discretized Stokes Equation}

The following benchmark deals with the safety analysis of a Stokes equation that describes the flow of an incompressible fluid in a two-dimensional spatial domain as described in [24]. The equations of the system are given by:

$$
\begin{aligned}
& \frac{\partial v}{\partial t}=\Delta v-\nabla \rho+f, \text { in } \Omega \times(0, T), \\
& \nabla v=0, \text { in } \Omega \times(0, T),
\end{aligned}
$$

where $v(\zeta, t) \in \mathbb{R}^{2}$ is the velocity vector, $\rho(\zeta, t) \in \mathbb{E}$ is the pressure, $f(\zeta, t) \in \mathbb{R}^{2}$ is the vector of external forces, $\Omega=[0,1] \times[0,1]$ is a square spatial domain, $T$ is the endpoint of the time interval, $\nabla$ denotes the divergence operator, and $\Delta=\nabla^{2}$. The stokes equations are parametrized using Dirichlet boundary conditions. Thus the velocity vanishes on the boundary of the domain. The equations are then semi-discretized over the spatial domain $\Omega$ using a uniform number of square cells and the well-known MAC scheme [20] (a scheme leveraging finite element methods) yielding an index-2 DAE with the following system matrices:

$$
E=\left[\begin{array}{cc}
I_{n_{v}} & 0 \\
0 & 0
\end{array}\right], A=\left[\begin{array}{cc}
A_{11} & A_{12} \\
A_{12}^{T} & 0
\end{array}\right], B=\left[\begin{array}{c}
B_{1} \\
B_{2}
\end{array}\right], x=\left[\begin{array}{c}
v_{h} \\
\rho_{h}
\end{array}\right],
$$


where $v_{h} \in \mathbb{R}^{n_{v}}$ and $\rho_{h} \in \mathbb{R}^{n_{\rho}}$ are the semi-discretized vectors of velocity and pressure, $A_{11} \in$ $\mathbb{R}^{n_{v} \times n_{v}}$ is the discrete Laplace operator, $A_{12} \in \mathbb{R}^{n_{v} \times n_{\rho}}$ is the gradient operator, and $A_{12}^{T} \in$ $\mathbb{R}^{n_{\rho} \times n_{v}}$ is the divergence operator. Furthermore, let $n$ be the number of discretized segments of the domain on the $x$ or $y$-axis, then the dimension of the DAE system is thus $3 n^{2}+2 n$. Thus by increasing the number of cells used to discretize the domain $\Omega$ we are able to obtain an index-2 DAE system with arbitrarily large dimension.

In this benchmark, we were primarily concerned with the velocity along the $x$ and $y$ axes, $v_{x}^{c}(t)$ and $v_{y}^{c}(t)$, of the fluid in the central cell of the spatial domain $\Omega$ and defined the unsafe set of the system to be Unsafe $\triangleq-v_{x}^{c}(t)-v_{y}^{c}(t) \leq 0.04$. We refer readers to the following paper [29] for a more detailed description of the decoupling and benchmark construction process for the Semi-discretized Stokes Equation.

\section{Reachability Analysis}

In this section we present a brief analysis of the RLC circuit benchmark in order to demonstrate the use of verification tools for index-1 DAEs. Although we have chosen to store all of our verification problems in a format used by the SpaceEx verification tool [15], only index-1 DAEs can be analyzed by the tools currently available in the research community. In order to construct the SpaceEx model file needed for verification for the RLC circuit benchmark, the DAE system given by (2.5) must be decoupled using the Marz decoupling method into an ODE subsystem coupled with algebraic constraints $[23,29]$. Once the DAE has been decoupled, we must ensure that the initial set of states and initial conditions are consistent and satisfy certain conditions. We refer the reader to the following paper for an in-depth discussion of these requirements $[23,29]$. The final step in generating the SpaceEx model file is the construction of an automaton

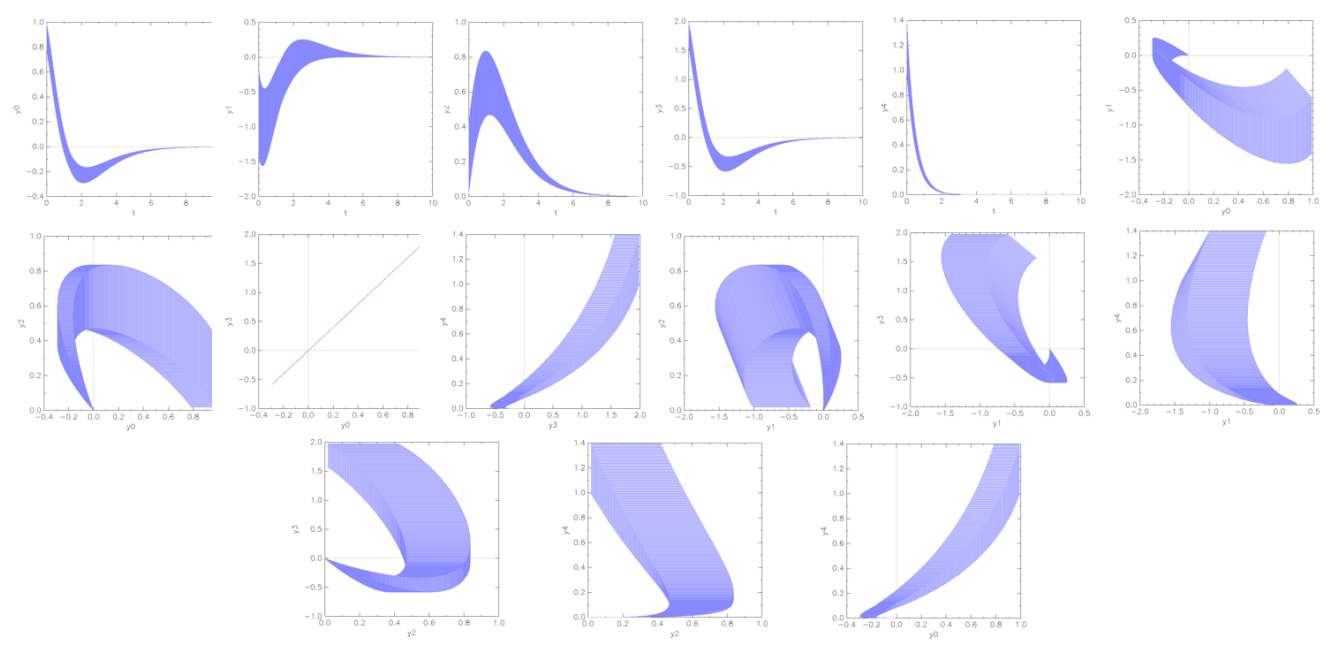

Figure 3.1: SpaceEx output for the RLC circuit benchmark reachable set over a ten second simulation

with a single mode whose continuous evolution is specified by the ODE subsystem and governed by a set of invariants corresponding to the algebraic constraints. The initial set of states used in this example can be found in the supplementary material included with this paper. In this example we perform the DAE decoupling, consistency checking, and SpaceEx model file 
generation using our tool Daev. We refer readers to the following paper [29] for an in-depth discussion our tool. Figure 3.1 displays the results of generating the reachable set for the RLC Circuit using SpaceEx over a ten second time period. Using this reachable set we can then reason about the overall safety of RLC circuit.

\section{Outlook}

In this manuscript we have presented a diverse set of challenging executable benchmarks for the verification of differential algebraic equations. We have also provided a tool named Daev that is able to print each benchmark in the SpaceEx format while ensuring the consistency of the initial sets and inputs of the benchmark problems. The problems detailed above range in dimensionality, DAE index, and come from various application domains in engineering and science. Despite the large number of CPS applications that involve high-index DAE dynamics, reachability analysis for DAE systems remains an open challenging problem that has not been adequately addressed in verification literature. To this end, the following paper seeks to stimulate the development of methodologies and research tools in the research community that can verify (or falsify) properties of safety critical DAE CPS. In future work, we wish to consider systems with index $\mu>3$ as well as consider non-linear differential algebraic equations.

\section{References}

[1] Althoff, M. An Introduction to CORA 2015. In ARCH14-15. 1st and 2nd International Workshop on Applied veRification for Continuous and Hybrid Systems (2015), G. Frehse and M. Althoff, Eds., vol. 34 of EPiC Series in Computing, EasyChair, pp. 120-151.

[2] Althoff, M., And Krogh, B. Reachability Analysis of Nonlinear Differential-Algebraic Systems. Automatic Control, IEEE Transactions on 59, 2 (2014), 371-383.

[3] Aström, K. J., And Murray, R. M. Feedback systems: An introduction for scientists and engineers. Tech. rep., 2004.

[4] BaK, S., And Duggirala, P. S. Simulation-equivalent Reachability of Large Linear Systems with Inputs. In International Conference on Computer Aided Verification (2017), Springer, pp. 401-420.

[5] Banagafya, N., Al, G., And Schilders, W. Index-aware Model Order Reduction Methods: Applications to Differential-Algebraic Equations, 1st ed. Atlantis Publishing Corporation, 2016.

[6] Brenan, K., Campbell, S., and Petzold, L. Numerical Solution of Initial-Value Problems in Differential-Algebraic Equations. Society for Industrial and Applied Mathematics, 1995.

[7] Byrne, G., And Ponzi, P. Differential-algebraic Systems, Their Applications and Solutions. vol. 12, pp. 377 - 382. XVIII Congress: The Use of Computers in Chemical Engineering.

[8] Chahlaoui, Y., And Van Dooren, P. A Collection of Benchmark Examples for Model Reduction of Linear Time Invariant Dynamical Systems.

[9] Chahlaoui, Y., And Van Dooren, P. Benchmark Examples for Model Reduction of Linear Time-Invariant Dynamical Systems. In Dimension Reduction of Large-Scale Systems (Berlin, Heidelberg, 2005), P. Benner, D. C. Sorensen, and V. Mehrmann, Eds., Springer Berlin Heidelberg, pp. 379-392.

[10] Chen, X., Ábrahám, E., and Sankaranarayanan, S. Flow*: an Analyzer for Non-linear Hybrid Systems. In International Conference on Computer Aided Verification (2013), Springer, pp. 258-263.

[11] Cross, E. A., And Mitchell, I. M. Level set methods for computing reachable sets of systems with differential algebraic equation dynamics. In 2008 American Control Conference (June 2008), pp. 2260-2265. 
[12] DAI, L. Singular control systems, vol. 118 of Lecture notes in control and information sciences. Springer, 1989.

[13] Dang, T., Donzé, A., And Maler, O. Verification of analog and mixed-signal circuits using hybrid system techniques. In Formal Methods in Computer-Aided Design (Berlin, Heidelberg, 2004), A. J. Hu and A. K. Martin, Eds., Springer Berlin Heidelberg, pp. 21-36.

[14] Duggirala, P. S., And Viswanathan, M. Parsimonious, Simulation Based Verification of Linear Systems. In International Conference on Computer Aided Verification (2016), Springer, pp. 477494.

[15] Frehse, G., Le Guernic, C., Donzé, A., Cotton, S., Ray, R., Lebeltel, O., Ripado, R., Girard, A., Dang, T., And Maler, O. SpaceEx: Scalable Verification of Hybrid Systems. In Computer Aided Verification (2011), Springer, pp. 379-395.

[16] Geerts, T. Solvability conditions, consistency, and weak consistency for linear differentialalgebraic equations and time-invariant singular systems: The general case. Linear Algebra and its Applications 181 (1993), 111 - 130.

[17] Gerdin, M. Parameter Estimation in Linear Descriptor Systems. Licentiate thesis no. 1085, Department of Electrical Engineering, Linköping University, SE-581 83 Linköping, Sweden, Apr. 2004.

[18] Girard, A. Reachability of uncertain linear systems using zonotopes. In Hybrid Systems: Computation and Control. Springer, 2005, pp. 291-305.

[19] Guernic, C. L., And Girard, A. Reachability Analysis of Linear Systems Using Support Functions. Nonlinear Analysis: Hybrid Systems 4, 2 (2010), 250-262.

[20] Han, H., ANd Wu, X. A new mixed finite element formulation and the mac method for the stokes equations. SIAM Journal on Numerical Analysis 35, 2 (1998), 560-571.

[21] Ho, C.-W., Ruehli, A., And Brennan, P. The Modified Nodal Approach to Network Analysis. IEEE Transactions on Circuits and Systems 22, 6 (Jun 1975), 504-509.

[22] Kunkel, P., And Mehrmann, V. L. Differential-Algebraic Equations: Analysis and Numerical Solution. European Mathematical Society, 2006.

[23] März, R. Canonical Projectors for Linear Differential Algebraic Equations. Computers and Mathematics with Applications 31, 4 (1996), 121 - 135. Selected Topics in Numerical Methods.

[24] Mehrmann, V., And Stykel, T. Balanced Truncation Model Reduction for Large-Scale Systems in Descriptor Form. In Dimension Reduction of Large-Scale Systems. Springer, 2005, pp. 83-115.

[25] Mitchell, I. M., And Susuki, Y. Level set methods for computing reachable sets of hybrid systems with differential algebraic equation dynamics. In Hybrid Systems: Computation and Control (Berlin, Heidelberg, 2008), M. Egerstedt and B. Mishra, Eds., Springer Berlin Heidelberg, pp. 630-633.

[26] Pasqualetti, F., Dorfler, F., And Bullo, F. Control-theoretic methods for cyberphysical security: Geometric principles for optimal cross-layer resilient control systems. IEEE Control Systems 35, 1 (Feb 2015), 110-127.

[27] Ruenli, A. Partial Element Equivalent Circuit (PEEC) Method and its Application in the Frequency and Time Domain. In Proceedings of Symposium on Electromagnetic Compatibility (Aug 1996), pp. 128-133.

[28] Schon, T., Gerdin, M., Glad, T., and Gustafsson, F. A Modeling and Filtering Framework for Linear Differential-algebraic Equations. In Decision and Control, 2003. Proceedings. 42nd IEEE Conference on (2003), vol. 1, IEEE, pp. 892-897.

[29] Tran, H.-D., Xiang, W., Hamilton, N., and Johnson, T. T. Simulation-Based Reachability Analysis for High-Index Large Linear Differential Algebraic Equations. ArXiv e-prints (Apr. 2018). 


\section{Appendix: Benchmark dimensionality, index and safety specifications}

Table 5.1: Overview of the dimensionality, index and safety specifications for the benchmark problems presented in this manuscript.

\begin{tabular}{lccc}
\hline Benchmarks & $\mathbf{n}$ & Index & Unsafe Set \\
\hline RL network [21] & 3 & 2 & $x_{1} \leq-0.2 \wedge x_{2} \leq-0.1$ \\
RL network [21] & 3 & 2 & $x_{1} \geq 0.2$ \\
\hline RLC circuit [12] & 4 & 1 & $x_{1}+x_{3} \geq 0.2$ \\
RLC circuit [12] & 4 & 1 & $x_{4} \leq-0.3$ \\
\hline Interconnected rotating & 4 & 2 & $x_{3} \leq-0.9$ \\
mass [28] & & & \\
Interconnected rotating & 4 & 2 & $x_{4} \leq-1.0$ \\
mass [28] & & & \\
\hline Generator [17] & 9 & 3 & $x_{9} \geq 0.01$ \\
Generator [17] & 9 & 3 & $x_{1} \geq 1.0$ \\
\hline Damped-mass spring [24] & 11 & 3 & $x_{3} \leq 1 \wedge x_{8} \leq 1.5$ \\
Damped-mass spring [24] & 11 & 3 & $x_{8} \leq-0.2$ \\
\hline PEEC [9] & 480 & 2 & $x_{478} \geq 0.05$ \\
PEEC [9] & 480 & 2 & $x_{478} \geq 0.01$ \\
\hline MNA-1 [9] & 578 & 2 & $x_{1} \geq-0.001$ \\
MNA-1 [9] & 578 & 2 & $x_{1} \geq-0.0015$ \\
\hline MNA-4 [9] & 980 & 3 & $x_{2} \geq 0.0005$ \\
MNA-4 [9] & 980 & 3 & $x_{2} \geq 0.0002$ \\
\hline Stokes-equation [24] & 4880 & 2 & $v_{x}^{c}+v_{y}^{c} \leq-0.04$ \\
Stokes-equation [24] & 4880 & 2 & $v_{x}^{c} \geq 0.2$ \\
\hline
\end{tabular}

